\title{
Neonatal suppurative submandibular sialadenitis
}

\author{
Dr. Narendra saini* ., Dr. Anamika Baghel \\ *Medical officer SCNU IGDH Seoni M.P,India.
}

\begin{abstract}
Submandibular suppurative sialadenitis occurring as an isolated lesion in the neonatal period is extremely rare. This report presents a rare case of isolated suppurative submandibular sialadenitis in a fullterm newborn without any risk factors. Possible etiology, diagnosis and management of this uncommon disease are discussed.
\end{abstract}

Keywords: newborn, submandibular, sialadenitis

\section{Introduction}

Neonatal sialadenitis of the submandibular gland is a very rare clinical entity. it almost always involves the parotid glands.(1) Infection of the sub-mandibular gland is rare(2).Information about the etiopathogenesis and management of the disease is very limited. Prematurity, prolonged gavage feeding and dehydration are the frequent causes. This report presents a rare case of isolated suppurative submandibular sialadenitis in a full-term newborn without any risk factors. Possible etiology, diagnosis and management of this uncommon disease are discussed.

\section{Case Presentation}

A 6-day-old male neonate presented with a one day history of swelling and redness of the submandibular region. She was born at 40 weeks of gestation with a birth weight of $2.6 \mathrm{~kg}$. The pregnancy was uncomplicated. The infant was breastfed and mother had no signs of mastitis.

on the day of admission a firm, tender, erythematous swelling about $2.4 \times 1.6 \mathrm{~cm}$ in diameter was noticed at the right submandibular region (Fig. 1). There was no evidence of erythema, swelling or tenderness in either parotid region. The infant was afebrile. Her vital parameters and hydration status were normal. Her examination was otherwise unremarkable.

\begin{tabular}{|l|l|}
\hline & $\begin{array}{l}\text { Fig 1: Neonate with submandibular swelling at the time of } \\
\text { admission }\end{array}$ \\
\hline
\end{tabular}

On admission, the total white blood cell count was $16,000 / \mathrm{mm}^{3}$ (69\% neutrophils, $25 \%$ lymphocytes, $4 \%$ monocytes, and 2\% stab). Erythrocyte sedimentation rate was $18 \mathrm{~mm} / \mathrm{hr}$ and Serum amylase was found within normal limit . Gram stain of the pus showed gram-positive cocci. A diagnosis of acute suppurative sialadenitis was established. After the pus and blood cultures were obtained, empiric intravenous treatment with ampicillin $(150 \mathrm{mg} / \mathrm{kg} / \mathrm{d})$ and gentamicin $(7.5 \mathrm{mg} / \mathrm{kg} / \mathrm{d})$ was initiated. Staphylococcus aureus was isolated from the purulent discharge whereas the neonate's blood cultures was sterile. gram stain and culture sensitivity report revealed staphylococcus aureus sensitive to cefotaxime and vancomycin. After admission swelling gradualy 
increase in size and undergo spontaneous rupture and drainage on day 9 and the palpable mass resolved . she was discharged with oral cepodoxime for 5 days. Follow-up examination demonstrated no residues or abnormalities of the gland..

\section{Discussion}

The first case of neonatal suppurative submandibular sialadenitis was reported by Schulman in 1950[3]. Submandibular sialadenitis usually follows infection of the parotid gland; submandibular gland infection without involvement of the parotid gland is seen infrequently. Infection of the submandibular gland is rare compared to the parotid gland because it produces more mucus, which is bacteriostatic, protecting the gland from infection[6].

The predisposing factors for suppurative sialadenitis in newborns are prematurity, dehydration, prolonged orogastric feeding and congenital anomalies of the floor of the mouth[7]. A clear association between prematurity and suppurative sialadenitis has been shown previously[ $[\mathbf{8}]$. Seventy-six percent of neonates with submandibular sialadenitis were born prematurely ( $\leq 35$ weeks). Prematurity has been described as the main risk factor for developing submandibular sialadenitis.

Dehydration and gavage feeding have been proposed as other predisposing factors, but neither of these was present in our case. Although dehydration has been implicated as a risk factor, signs of dehydration may not be observed. Only a few neonates with submandibular sialadenitis were not gavage-fed[프. Transmission of bacteria during breastfeeding or through contaminated formula can be a potential cause of sialadenitis. In the presented case, bacterial colonization of the bottle-fed mother's milk with S. aureus could have occurred during storage or warming before feeding. The infant was given breast-milk with a bottle a few times, but the mother had no signs of mastitis and no bacterial growth was observed in her milk.

Staphylococcus aureus is the usual causative organism in neonatal sialadenitis. The other isolated organisms have included streptococci, Pseudomonas aeruginosa, Escherichia coli and Moraxella catarrhalis. Anaerobic bacteria have been recovered from salivary gland infections in older children and adults[9], but Prevotella species (intermedia/melaninogenica), Fusobacterium nucleatum, and Peptostreptococcus magnus have recently been reported in two newborns[4]. Suppurative sialadenitis due to methicillinresistant S. aureus (MRSA) has also been described[7].

The diagnosis of submandibular sialadenitis can be made on clinical grounds. However, systemic manifestations may be minimal in neonates with salivary gland infection[6]. The temperature elevation may be slight and the infants may continue to feed well[11].

The administration of antimicrobial therapy is an essential part of the management of patients with suppurative sialadenitis. Most cases respond to antimicrobial therapy; however, sometimes abscess formation requires surgical drainage[10,11]. Empirical antibiotics for sialadenitis in the newborn should cover both grampositive and gram-negative organisms. Although S. aureus is the most common responsible organism in neonatal sialadenitis, Escherichia coli, Pseudomonas aeruginosa, and Neisseria catarrhalis have been reported as the other causative agents[]].

\section{Conclusion}

Isolated neonatal suppurative sialadenitis is rare, it should be suspected even in full-term infants presenting with an erythematous submandibular mass without any predisposing factors.

\section{Reference}

[1]. Greenberg MS. Salivary gland disease. In: Burkets Oral Medicine-Diagnosis and Treat-ment, 9th edn. Eds. Lynch MA, Brightman VJ, Greenberg MS. Philadelphia, J.B. Lippinott Co, 1994; pp 415-431.

[2]. Ungkanont K, Kolatat T, Tantinikorn W. Neonatal suppurative submandibular sialade-nitis: A rare clinical entry. Int J Pediatr Otorhinolaryngol 1998; 43:141-145.

[3]. Schulman BH. Acute suppurative infections of the salivary glands in the newborn. Am J DisChild1950;80:413-416.

[4]. Brook I. Suppurative sialadenitis associated with anaerobic bacteria in newborns. Pediatr InfectDisJ2006;25:280.

[5]. Weibel L, Goetschel P, Meier R, Radivojevic V, Berger C. Neonatal suppurative submandibularsialadenitis.PediatrInfectDisJ2005;24:379-381.

[6]. Leake D, Leake R. Neonatal suppurative parotitis. Pediatrics 1970; 46: 202-207.

[7]. McAdams RM, Mair EA E, Rajnik M. Neonatal suppurative submandibular sialadenitis: case report and literature review. Int J Pediatr Otorhinolaryngol 2005; 69: 993-997.

[8]. Garavello W, Romagnoli M, Somigliana E, Zorloni C, Sordo L, Gaini RM. The intriguing association between prematurity and neonatal isolated submandibular suppurative sialadenitis. Int J Pediatr Otorhinolaryngol 2002; 62: 41-44.

[9]. Brook I. Aerobic and anaerobic microbiology of suppurative sialadenitis. J Med Microbiol 2002;51:526-529.

[10]. Bova R, Walker P. Neonatal submandibular sialadenitis progressing to submandibular gland abscess. Int J Pediatr Otorhinolaryngol 2000; 53: 73-75.

[11]. Bafaqeeh SA. Complicated neonatal submandibular suppurative sialadenitis. Int J Pediatr Otorhinolaryngol 1998; 44: $267-271$. 